\title{
Dynamics of spatially homogeneous solutions of the Einstein-Vlasov equations which are locally rotationally symmetric
}

\author{
A D Rendall $\dagger$ and K P Tod $\ddagger$ \\ $\dagger$ Max-Planck-Institut für Gravitationsphysik, Schlaatzweg 1, 14473 Potsdam, Germany \\ \$ University of Oxford, Mathematical Institute, 24-29 St Giles, Oxford OX1 3LB, UK
}

Received 3 December 1998, in final form 2 March 1999

\begin{abstract}
The dynamics of a class of cosmological models with collisionless matter and four Killing vectors is studied in detail and compared with that of corresponding perfect fluid models. In many cases it is possible to identify asymptotic states of the spacetimes near the singularity or in a phase of unlimited expansion. Bianchi type II models show oscillatory behaviour near the initial singularity which is, however, simpler than that of the mixmaster model.
\end{abstract}

PACS numbers: 0420D, 9880

\section{Introduction}

In studies of the dynamics of spatially homogeneous cosmological models it is usual to choose a perfect fluid with a linear equation of state to describe the matter. The book [19] provides an excellent guide to the subject. In view of the fact that this restriction is made so frequently in the literature, it is natural to pose the question: to what extent the conclusions obtained would change if the matter model were chosen differently? In [14] it was shown that in the case of collisionless matter described by the Vlasov equation significant changes can occur in comparison with the case of a perfect fluid. More specifically, it was shown that a solution of Bianchi type I exists whose qualitative behaviour near the initial singularity is different from that of any spacetime of that Bianchi type whose matter content is a fluid with a physically reasonable equation of state, linear or nonlinear. In the following this analysis will be generalized to show just how different models with collisionless matter can be from models with a perfect fluid having the same symmetry. Differences are found in models of Bianchi type II (theorem 4.2), Bianchi type III (theorem 5.2) and Kantowski-Sachs models (theorem 5.1). These concern both the initial singularity and phases of unlimited expansion. Perhaps the most striking case is that of the initial singularity in the Bianchi type II models, where we find persistent oscillatory behaviour near the singularity. This is quite different from the known behaviour of type II perfect fluid models.

The significance of the choice of matter model in cosmology can be illustrated by considering the radiation-dominated era of the early universe. Here the universe is filled with particles, which either are massless (e.g. photons) or have such high energies that they can plausibly be treated as massless. This situation can be described by kinetic theory with massless particles. If the matter distribution is isotropic then this is equivalent to a radiation 
fluid. However, once isotropy is abandoned this is no longer the case. Since the results of this paper show that the dynamics can change drastically when the kinetic description is replaced by an effective fluid description, it is necessary to think carefully about the choice of matter model as soon as anisotropy is allowed. Note that this discussion does not depend on the particular choice of a Vlasov model, but applies equally to a (presumably more realistic) description by means of the Boltzmann equation with a non-vanishing collision term.

Our results will also illuminate another matter. In [11] Lukash and Starobinski gave a heuristic analysis of a locally rotationally symmetric (LRS) model of Bianchi type I with collisionless matter consisting of massless particles. Their conclusion was that in the expanding direction the model would isotropize so that at large times it would look like a FriedmanRobertson-Walker model. On the one hand, we are able to prove rigorously that the heuristic analysis of [11] gives the correct result; on the other hand, we show that this result depends essentially on the assumption of a symmetry of Bianchi type I. If this symmetry type is replaced by Bianchi type II (keeping the LRS assumption and massless collisionless particles) then the anisotropy tends to a constant non-zero value at large times.

The cosmological models studied in this paper are LRS spatially homogeneous spacetimes with matter described by the Vlasov equation for massless particles. The reason for imposing the LRS condition is that it allows the Vlasov equation to be solved explicitly so that the Einstein-Vlasov equations reduce to a system of ordinary differential equations, albeit with coefficients, which are not explicitly known and depend on the chosen initial data. The reason for choosing the particles to be massless is that this allows a reduction of the system of ordinary differential equations (ODEs) similar to that carried out for perfect fluids with a linear equation of state by Wainwright and Hsu [20]. It has not proved possible to analyse the global behaviour of solutions to our system of ODEs completely. However, a number of partial results have been obtained, which show that there is considerable variety in the asymptotic behaviour of solutions near an initial singularity or during a phase of unlimited expansion. In particular, the reflection-symmetric LRS Bianchi type I solutions with massless particles are analysed completely with respect to their asymptotic behaviour, thus improving markedly on the results obtained on that class of spacetimes in [14].

The matter model used in the following will now be described. The matter consists of particles of zero rest mass, which propagate through spacetime freely without collisions. Each particle is affected by the others only by the gravitational field, which they generate collectively. The wordline of each particle is a null geodesic. Each geodesic has a natural lift to the tangent bundle of spacetime. Thus the geodesic equation defines a flow on the tangent bundle. By means of the metric this may if desired be transported to the cotangent bundle and here it will be convenient to do so. The subset of the cotangent bundle consisting of all covectors obtained by lowering the index of future-pointing null vectors, which will be denoted by $P$, is invariant under the flow and thus the flow may be restricted to it. The basic matter field used to describe the collisionless particles is a non-negative real-valued function $f$ on $P$ which represents the density of particles with given position and momentum at a given time. Choosing appropriate coordinates $x^{\alpha}$ on spacetime and letting $\left(x^{\alpha}, p_{\alpha}\right)$ be the corresponding coordinates on the cotangent bundle, the manifold $P$ can be coordinatized by $\left(x^{\alpha}, p_{a}\right)$. Here the convention is used that Greek and Roman indices run from 0 to 3 and 1 to 3, respectively. We write $t$ for $x^{0}$ and it is assumed that $t$ increases towards the future. The field equation for $f$, the Vlasov equation, says geometrically that $f$ is constant along the geodesic flow. In the coordinates just introduced its explicit form is

$$
\partial f / \partial t+\left(p^{a} / p^{0}\right) \partial f / \partial x^{a}+\left(\Gamma_{b \gamma}^{\alpha} p_{\alpha} p^{\gamma} / p^{0}\right) \partial f / \partial p_{b}=0
$$

where $p^{0}$ is to be determined from $p^{a}$ by the relation $g_{\alpha \beta} p^{\alpha} p^{\beta}=0$ and indices are raised and 
lowered using the spacetime metric $g_{\alpha \beta}$ and its inverse. In order to couple the Vlasov equation to the Einstein equation, it is necessary to define the energy-momentum tensor. It is given by

$$
T_{\alpha \beta}=-\int f p_{\alpha} p_{\beta}|g|^{-1 / 2} / p_{0} \mathrm{~d} p_{1} \mathrm{~d} p_{2} \mathrm{~d} p_{3} .
$$

In fact, for Bianchi models it is more useful to replace the coordinate components of the momentum used in these equations by components in a suitable frame. The only change in the equations is that the Christoffel symbols in the Vlasov equation are replaced by the connection coefficients in the given frame. For more information about the Vlasov equation in general relativity the reader is referred to $[6,15]$.

Spatially homogeneous spacetimes fall into three broad classes, known as Bianchi class A, Bianchi class B and Kantowski-Sachs (see [19]). Each of the two Bianchi classes can be further divided into Bianchi types. A spatially homogeneous spacetime in one of the Bianchi classes is called locally rotationally symmetric if it has, in addition to the three Killing vector fields needed for spatial homogeneity, a fourth one. This can only happen for certain symmetry types. In class A the Bianchi types, which allow an LRS special case are I, II, VII 0 , VIII and IX. In class B it is types III, V and $\mathrm{VII}_{h}$ which allow this [12]. The Kantowski-Sachs spacetimes automatically have a fourth Killing vector. There exist solutions of the EinsteinVlasov equations with $k=-1$ Robertson-Walker symmetry and these have, in particular, Bianchi type $\mathrm{V}$ and Bianchi type $\mathrm{VII}_{h}$ symmetry with any non-zero $h$. We did not attempt to ascertain whether there are other examples of solutions of these Bianchi types with LRS symmetry, and these types are not considered further in this paper. A spatially homogeneous solution of the Einstein-Vlasov equations has by definition the property that both the geometry and the phase space density of particles are invariant under the group action defining the symmetry type. A similar remark applies to an additional LRS symmetry. It would be nice if the invariance of $f$ under the group in a Bianchi model could be expressed by the condition that $f$ depends only on time and momentum when expressed with respect to a left-invariant frame on the group defining the symmetry. Unfortunately, as discussed in [13], this does not work in general. It does work for all LRS Bianchi models of class A and type III and for Kantowski-Sachs models [12]. This is the reason why LRS models are relatively tractable. In the following we consider LRS models, which are of Kantowski-Sachs type, or of Bianchi type I, II, III, VII 0 , VIII or IX.

In the next section it is shown how in the class of spacetimes of interest the EinsteinVlasov equations with given initial data can be reduced to a system of ordinary differential equations. In fact, two systems are needed. The first includes the solutions of types I, II, $\mathrm{VII}_{0}$, VIII and IX, while the second includes those of types I and III and the Kantowski-Sachs models. Note that the solutions of type I are represented in both systems and understanding the Bianchi I case is central to analysing the general case. The analysis of the Bianchi I system is carried out in section 3 . This is then used in sections 4 and 5 to obtain results on the first and second systems of ODEs, respectively. In the last section the results are summarized and their wider significance is examined. An appendix collects together some results from the theory of dynamical systems used in the body of the paper.

\section{Reduction to an ODE problem}

In a spacetime with Bianchi symmetry the metric can be written in the form

$$
\mathrm{d} s^{2}=-\mathrm{d} t^{2}+g_{a b}(t) \theta^{a} \otimes \theta^{b}
$$

where $\left\{\theta^{a}\right\}$ is a left-invariant coframe on the Lie group $G$ which defines the symmetry. The particular Bianchi type is determined by the structure constants of the Lie algebra of $G$. The 
extra symmetry, which is present in the LRS case implies that the metric $g_{a b}(t)$ is diagonal, with two of the diagonal elements being equal [12]. Thus (3) simplifies to

$$
\mathrm{d} s^{2}=-\mathrm{d} t^{2}+a^{2}(t)\left(\theta^{1}\right)^{2}+b^{2}(t)\left(\left(\theta^{2}\right)^{2}+\left(\theta^{3}\right)^{2}\right)
$$

for two functions $a(t)$ and $b(t)$ of one variable. If $k^{\alpha}$ is any Killing vector field then the function $p_{\alpha} k^{\alpha}$ on the cotangent bundle is constant along geodesics and hence satisfies the Vlasov equation. Any function of quantities of this type for different Killing vectors also satisfies the Vlasov equation. The Killing vectors on a spacetime with Bianchi symmetry include those defined by right-invariant vector fields on the Lie group $G$, but the result of evaluating a left-invariant 1 -form on one of these is not in general constant. Thus we cannot simply solve the Vlasov equation by choosing an arbitrary function of the components $p_{a}$ with respect to a left-invariant basis. However, for the LRS spacetimes of Bianchi class A or type III considered here a function of the form $f\left(t, p_{1}, p_{2}, p_{3}\right)=f_{0}\left(p_{1}, p_{2}^{2}+p_{3}^{2}\right)$ does satisfy the Vlasov equation and, in fact, is the most general solution with the full LRS symmetry [12]. Here $p_{1}, p_{2}$ and $p_{3}$ are the components of the momentum in the coframe $\left\{\theta^{a}\right\}$. Since $f$ does not depend explicitly on time in this representation, the function $f_{0}$ can be identified with the initial datum for the solution of the Vlasov equation at a fixed time. A similar statement holds for Kantowski-Sachs spacetimes. The metric can be written in the form (4) where $\theta^{1}$ is invariant under the symmetry group and $\theta^{2}$ and $\theta^{3}$ make up any (locally defined) orthonormal coframe on the 2 -sphere. The expression $p_{2}^{2}+p_{3}^{2}$ is not changed by a change in orthonormal coframe and so it makes sense to consider the above form of $f$ in terms of $f_{0}$ in Kantowski-Sachs spacetimes as well. If $f$ is of this form it satisfies the Vlasov equation. Thus the Vlasov equation has been solved explicitly in the class of spacetimes to be studied. It remains to determine the form of the Einstein equations. In fact, one further restriction will be imposed. The distribution function given above is automatically an even function of $p_{2}$ and $p_{3}$. However, it need not be even in $p_{1}$. If it is even in $p_{1}$ we say, as in [14], that the solution is reflection symmetric. Only reflection-symmetric solutions will be considered in the following. For convenience we say that a function of $p_{1}, p_{2}$ and $p_{3}$ which depends only on $p_{1}$ and $p_{2}^{2}+p_{3}^{2}$ and which is even in $p_{1}$ has special form.

If the Einstein equations are split as usual into constraints and evolution equations then it turns out that in this class of spacetimes the momentum constraint is automatically satisfied. Only the Hamiltonian constraint and the evolution equations are left. The former is an algebraic relation between $a, b$ and their time derivatives $\mathrm{d} a / \mathrm{d} t, \mathrm{~d} b / \mathrm{d} t$. The latter provide ordinary differential equations for the evolution of $a$ and $b$ which are second order in time. It will be convenient to write these equations in terms of some alternative variables. Consider first the mean curvature of the homogeneous hypersurfaces:

$$
\operatorname{tr} k=-\left[a^{-1} \mathrm{~d} a / \mathrm{d} t+2 b^{-1} \mathrm{~d} b / \mathrm{d} t\right] .
$$

Note that $\operatorname{tr} k$ is minus the expansion of the normal vector field to the homogeneous hypersurfaces. Thus tr $k<0$ corresponds to an expanding cosmological model. A new time coordinate $\tau$ can be defined by $\tau(t)=-\int_{t_{0}}^{t} \operatorname{tr} k(t) \mathrm{d} t$ for some arbitrary fixed time $t_{0}$. In the following a dot over a quantity denotes its derivative with respect to $\tau$. Now define

$$
\begin{aligned}
& r=b / a, \\
& N_{1}=-\epsilon_{1}\left(a / b^{2}\right)(\operatorname{tr} k)^{-1}, \\
& N_{2}=-\epsilon_{2} a^{-1}(\operatorname{tr} k)^{-1}, \\
& \Sigma_{+}=-3\left(b^{-1} \mathrm{~d} b / \mathrm{d} t\right)(\operatorname{tr} k)^{-1}-1, \\
& B=-b^{-1}(\operatorname{tr} k)^{-1}
\end{aligned}
$$


where $\epsilon_{1}$ and $\epsilon_{2}$ will be $-1,0$ or 1 , depending on the symmetry type considered. The variables $N_{1}, N_{2}$ and $\Sigma_{+}$are closely related to the variables of the same names used by Wainwright and Hsu [20]. (Note that we adopt the conventions of [20] rather than those of [19], which differ by a factor of three in some places.)

Two systems of ODEs will now be considered, which between them are equivalent to the evolution part of the Einstein-Vlasov equations for all the relevant symmetry types.

The first system is

$\dot{r}=\Sigma_{+} r$

$\dot{N}_{1}=\left[-\frac{1}{4} N_{1}\left(N_{1}-4 N_{2}\right)+\frac{1}{3}\left(1-4 \Sigma_{+}+\Sigma_{+}^{2}\right)\right] N_{1}$

$\dot{N}_{2}=\left[-\frac{1}{4} N_{1}\left(N_{1}-4 N_{2}\right)+\frac{1}{3}\left(1+2 \Sigma_{+}+\Sigma_{+}^{2}\right)\right] N_{2}$

$\dot{\Sigma}_{+}=\frac{3}{2}\left\{\frac{1}{2} N_{1}^{2}+\frac{1}{6} N_{1}\left(N_{1}-4 N_{2}\right)\left(1-2 \Sigma_{+}\right)\right.$

$$
\left.+\left[-\frac{1}{4} N_{1}\left(N_{1}-4 N_{2}\right)+\frac{1}{3}\left(1-\Sigma_{+}^{2}\right)\right]\left[\frac{1}{3}\left(1-2 \Sigma_{+}\right)-Q\right]\right\} .
$$

Here $Q$ is defined to be

$$
Q(r)=r^{2}\left[\frac{\int f_{0}\left(p_{i}\right) p_{1}^{2}\left(r^{2} p_{1}^{2}+p_{2}^{2}+p_{3}^{2}\right)^{-1 / 2} \mathrm{~d} p_{1} \mathrm{~d} p_{2} \mathrm{~d} p_{3}}{\int f_{0}\left(p_{i}\right)\left(r^{2} p_{1}^{2}+p_{2}^{2}+p_{3}^{2}\right)^{1 / 2} \mathrm{~d} p_{1} \mathrm{~d} p_{2} \mathrm{~d} p_{3}}\right]
$$

where $f_{0}$ is a fixed smooth function of special form and compactly supported on $\mathbb{R}^{3}$. The Hamiltonian constraint is

$$
16 \pi \rho /(\operatorname{tr} k)^{2}=-\frac{1}{2} N_{1}\left(N_{1}-4 N_{2}\right)+\frac{2}{3}\left(1-\Sigma_{+}^{2}\right)
$$

where $\rho$ is the energy density and to take account of the positivity of $\rho$, only the region satisfying the inequality

$$
-\frac{1}{2} N_{1}\left(N_{1}-4 N_{2}\right)+\frac{2}{3}\left(1-\Sigma_{+}^{2}\right) \geqslant 0
$$

is considered. The quantity on the left-hand side of (9) corresponds to $\frac{3}{2} \Omega$, where $\Omega$ is the density parameter often used in cosmology. Define submanifolds of this region by the following conditions:

$$
\begin{aligned}
& S_{1}: \quad N_{1}=N_{2}=0 \\
& S_{2}: \quad N_{1} \neq 0, \quad N_{2}=0 \\
& S_{3}: \quad N_{1}=0, \quad N_{2} \neq 0 \\
& S_{4}: \quad N_{1} \neq 0, \quad N_{2} \neq 0, \quad N_{2}=-r^{2} N_{1} \\
& S_{5}: \quad N_{1} \neq 0, \quad N_{2} \neq 0, \quad N_{2}=r^{2} N_{1} \text {. }
\end{aligned}
$$

The submanifolds $S_{1}, S_{2}, S_{3}, S_{4}$ and $S_{5}$ correspond to Bianchi types I, II, VII 0 , VIII and IX, respectively. To make the correspondence with spacetime quantities in these different cases $\left(\epsilon_{1}, \epsilon_{2}\right)$ should be chosen to be $(0,0),(1,0),(0,1),(-1,1)$ and $(1,1)$, respectively. Note that if $r$ is replaced by $\tilde{r}=r^{-1}$ in (7) an almost identical system is obtained, with the sign in the first equation being reversed.

The second system is

$$
\begin{aligned}
& \dot{r}=\Sigma_{+} r \\
& \dot{B}=\left[\epsilon B^{2}+\frac{1}{4}+\frac{1}{12}\left(1-2 \Sigma_{+}\right)^{2}\right] B \\
& \dot{\Sigma}_{+}=\frac{3}{2}\left\{-\frac{2}{3} \epsilon B^{2}\left(1-2 \Sigma_{+}\right)+\left[\epsilon B^{2}+\frac{1}{3}\left(1-\Sigma_{+}^{2}\right)\right]\left[\frac{1}{3}\left(1-2 \Sigma_{+}\right)-Q\right]\right\}
\end{aligned}
$$

where $\epsilon$ belongs to the set $\{-1,0,1\}$. Only the region satisfying the inequality

$$
2 \epsilon B^{2}+\frac{2}{3}\left(1-\Sigma_{+}^{2}\right) \geqslant 0
$$


is considered. The cases $\epsilon=-1,0$ and 1 correspond to Bianchi type III, Bianchi type I and Kantowski-Sachs, respectively. Note that the restriction of the system (7) to $S_{1}$ is identical to the system consisting of the first and third equations of (11) for $\epsilon=0$. This restricted system will be referred to in the following as the Bianchi I system. It was introduced in section 6 of [14] with slightly different variables.

If a solution of (7) and a fixed $f_{0}$ are given, it is possible to construct a spacetime as follows. Suppose that $\tau=0$ is contained in the domain of definition of the solution. Since the system is autonomous this is no essential restriction. Choose a negative number $H_{0}$. Define

$$
\rho=(1 / 16 \pi) H_{0}^{2}\left[-\frac{1}{2} N_{1}(0)\left(N_{1}(0)-4 N_{2}(0)\right)+\frac{2}{3}\left(1-\Sigma_{+}^{2}(0)\right)\right] .
$$

Let

$$
I=\int f_{0}\left(p_{i}\right)\left[(r(0))^{2} p_{1}^{2}+p_{2}^{2}+p_{3}^{2}\right]^{1 / 2} \mathrm{~d} p_{1} \mathrm{~d} p_{2} \mathrm{~d} p_{3}
$$

and define

$$
\begin{aligned}
& a_{0}=\rho^{-1 / 4} I^{1 / 4}(r(0))^{-3 / 4} \\
& b_{0}=\rho^{-1 / 4} I^{1 / 4}(r(0))^{1 / 4} .
\end{aligned}
$$

In terms of these quantities we can define an initial metric by

$$
a_{0}^{2}\left(\theta^{1}\right)^{2}+b_{0}^{2}\left(\left(\theta^{2}\right)^{2}+\left(\theta^{3}\right)^{2}\right) .
$$

Similarly, we can define an initial second fundamental form by

$$
-\frac{1}{3}\left(1-2 \Sigma_{+}(0)\right) H_{0} a_{0}^{2}\left(\theta^{1}\right)^{2}+\frac{1}{3}\left(1+\Sigma_{+}(0)\right) H_{0} b_{0}^{2}\left(\left(\theta^{2}\right)^{2}+\left(\theta^{3}\right)^{2}\right) .
$$

These data satisfy the constraints by construction. Consider now the spacetime which evolves from these initial data. It is of the form (3). For the Einstein-Vlasov system in a spacetime of the form (3) with a fixed time-independent distribution function is a system of second-order ODEs which has solutions corresponding to data for $(a, b, \mathrm{~d} a / \mathrm{d} t, \mathrm{~d} b / \mathrm{d} t)$. These data can be chosen so as to reproduce the data of interest for the Einstein-Vlasov system by choosing $\mathrm{d} a / \mathrm{d} t=\frac{1}{3}\left(1-2 \Sigma_{+}(0)\right) H_{0} a$ and $\mathrm{d} b / \mathrm{d} t=-\frac{1}{3}\left(1+\Sigma_{+}(0)\right) H_{0} b$ for $t=t_{0}$. This spacetime defines a solution of (7) via (6). (Note that $t=t_{0}$ corresponds to $\tau=0$.) Thus the two solutions are identical. In this way a spacetime has been constructed, which gives rise to the solution of (7) we started with. This spacetime may be obtained more explicitly if desired. In order to do this, first solve the equation

$$
\partial_{\tau}(\operatorname{tr} k)=-\left[-\frac{1}{4} N_{1}\left(N_{1}-4 N_{2}\right)+\frac{1}{3}\left(2+\Sigma_{+}^{2}\right)\right] \operatorname{tr} k
$$

with initial data $H_{0}$. Then $\rho$ can be obtained from the Hamiltonian constraint (9). The definition of $\rho$ in terms of $f_{0}$ can then be combined with $r$ to give $a$ and $b$ as in (15). Finally, $t$ can be obtained from $\operatorname{tr} k$. All the considerations here in the case of (7) are equally applicable in the case of (11). The analogue of equation (18) is

$$
\partial_{\tau}(\operatorname{tr} k)=-\left[\epsilon B^{2}+\frac{1}{3}\left(2+\Sigma_{+}^{2}\right)\right] \operatorname{tr} k
$$

Solutions of the Einstein equations with matter described by a perfect fluid with a linear equation of state $p=(\gamma-1) \rho$ which belong to one of the symmetry types studied in the case of collisionless matter in the following can be described by equations very similar to (7) and (11). The similarity is particularly great in the case where $\gamma=\frac{4}{3}$ (radiation fluid). In that case the only difference is that the function $Q(r)$ should be replaced by the constant value $\frac{1}{3}$. This leads to a decoupling of the first equation in each system, so that it is possible to restrict attention to the remaining equations when investigating the dynamics. (This last remark also 
applies to the system obtained for other values of $\gamma$.) The equation for $r$ can be integrated afterwards if desired.

In [14] it was proved that $Q(r)$ as defined in (8) tends to zero as $r$ tends to zero and that if $Q(0)$ is defined to be zero the resulting extension of $Q$ is $C^{1}$ with $Q^{\prime}(0)=0$. This means, in particular, that the dynamical system (7) has a well defined $C^{1}$ extension to $r=0$. In a similar way it can be shown that if a function $\tilde{Q}$ is defined by $\tilde{Q}(\tilde{r})=Q(r)$ then $\tilde{Q}$ can be extended in a $C^{1}$ manner to $\tilde{r}=0$ in such a way that $\tilde{Q}(0)=1$ and $\tilde{Q}^{\prime}(0)=0$. For $1-\tilde{Q}=\left(\rho-T_{1}^{1}\right) / \rho=2 T_{2}^{2} / \rho$ and this last expression is $\mathrm{O}\left(\tilde{r}^{4 / 3}\right)$ as $\tilde{r} \rightarrow 0$ by lemma 4.2 of [14]. By using a coordinate $\hat{r}=r /(r+1)$ it is possible to map the system (7) with $r$ ranging from zero to infinity onto a region with $\hat{r}$ ranging from zero to one. Moreover, the system extends in a $C^{1}$ manner to the boundary components $\hat{r}=0$ and 1 . The coordinate $\hat{r}$ has been introduced purely to demonstrate that the system (7) can be smoothly compactified in the $r$-direction. For computations it is more practical to use the local coordinates $r$ and $\tilde{r}$. In particular, these considerations allow us to regard the Bianchi I system as being defined on a compact set.

The compactification of the system (7) is defined on a region with a boundary. Different parts of the boundary are given by $\hat{r}=0,1$ and the case of equality in (10). The complement of the boundary will be called the interior region in the following. A solution which lies in the interior region corresponds to a smooth non-vacuum solution of the Einstein-Vlasov equations. A solution which lies in the part of the boundary where (10) becomes an equality corresponds to a solution of the vacuum Einstein equations. A solution which lies in the part of the boundary given by $\hat{r}=0$ or 1 corresponds to a distributional solution of the Einstein-Vlasov equations, as will be explained in more detail below. The system (11) can be compactified in a way very similar to that taken in the case of (7). The comments on the interpretation of different types of solutions of the compactification of (7) just made also apply to the compactification of (11), with (10) being replaced by (12).

Consider now the stationary points of the system (7), or rather of its compactification. (This distinction will not always be made explicitly in what follows.) In section 4 it will be shown that all stationary points where $r$ has a finite non-zero value belong to the subset $S_{1}$ corresponding to solutions of type I. In particular, they correspond to stationary points of the Bianchi I system, which will be studied in detail in the next section.

\section{The Bianchi I system}

It turns out that the Bianchi I system plays a central role in the dynamics of solutions of the systems (7) and (11). In this section the asymptotic behaviour of solutions of this system is determined, both for $\tau \rightarrow-\infty$ (approach to the singularity) and for $\tau \rightarrow \infty$ (unlimited expansion).

The first step in analysing the Bianchi I system is to determine the stationary points. This can be done using the fact, proved in [14], that $Q$ is strictly monotonic for $r>0$ so that there is a unique $r_{0}$ with $Q\left(r_{0}\right)=\frac{1}{3}$. With this information it is straightforward to show that the coordinates of the stationary points in the $\left(r, \Sigma_{+}\right)$-plane are $\left(r_{0}, 0\right),(0,-1),\left(0, \frac{1}{2}\right)$, $(0,1),(\infty,-1)$ and $(\infty, 1)$. Here $r=\infty$ is to be interpreted as $\tilde{r}=0$ or $\hat{r}=1$. Call these points $P_{1}, \ldots, P_{6}$, respectively (see figure 1 ). The next step is to linearize the system about the stationary points. Recall that a stationary point is called hyperbolic if none of its eigenvalues are purely imaginary. In the following we call a stationary point degenerate if it is not hyperbolic. The point $P_{1}$ is a hyperbolic sink, while $P_{4}$ is a hyperbolic source. The points $P_{2}, P_{3}$ and $P_{6}$ are hyperbolic saddles, while $P_{5}$ is degenerate, with one zero eigenvalue. 


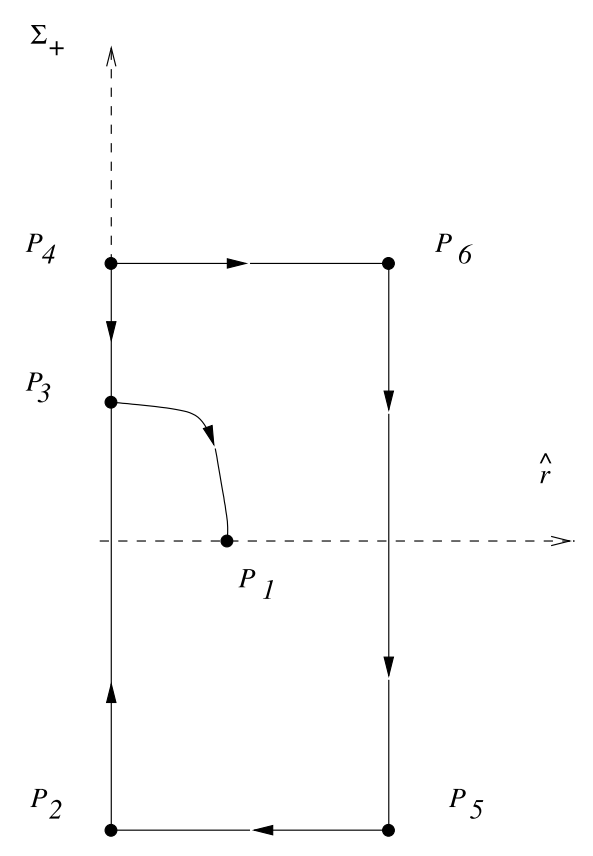

Figure 1. The $\left(\hat{r}, \Sigma_{+}\right)$-plane and the fixed points for Bianchi type I. (Here, and in figures 2 and 3 , coordinate axes, which are not trajectories of the system are shown as broken lines.)

Before proceeding further, we state the main result of this section.

Theorem 3.1. If a smooth non-vacuum reflection-symmetric LRS solution of Bianchi type I of the Einstein-Vlasov equations for massless particles is represented as a solution of (7) with $N_{1}=N_{2}=0$ then for $\tau \rightarrow \infty$ it converges to the point $P_{1}$. For $\tau \rightarrow-\infty$ either

(a) it converges to $P_{1}$ and in that case it stays for all time at the point $P_{1}$ or

(b) it converges to the point $P_{3}$ and it belongs to the unstable manifold of $P_{3}$ or

(c) it converges to $P_{4}$.

All of these cases occur, and (c) is the generic case in the sense that it occurs for an open dense set of initial data.

This will be proved in a series of lemmas. Terminology from the theory of dynamical systems which may be unfamiliar to the reader is explained in the appendix.

Lemma 3.1. If a solution of the Bianchi I system in the interior enters the region $\Sigma_{+}>\frac{1}{2}$ then for $\tau \rightarrow-\infty$ it belongs to case (c) of theorem 3.1. A solution of the Bianchi I system in the interior has no $\omega$-limit points with $\Sigma_{+} \geqslant \frac{1}{2}$.

Proof. A solution of the Bianchi I system satisfies $\dot{\Sigma} \leqslant-\frac{1}{2}\left(1-\Sigma_{+}^{2}\right) Q$ when $\Sigma_{+}>\frac{1}{2}$ and so for any solution which enters the given region, $\Sigma_{+}$is non-decreasing towards the past and it is in the region for all earlier times. If $\Sigma_{+}$did not tend to 1 as $\tau \rightarrow-\infty$ then we would have $\dot{\Sigma}_{+} \leqslant-C<0$ at early times, a contradiction. Once we know that $\Sigma_{+} \rightarrow 1$ as $\tau \rightarrow-\infty$ it follows immediately that $r \rightarrow 0$. Thus the solution converges to $P_{4}$. Consider now the forward time direction. Since $\Sigma_{+}$is positive, $r$ is increasing. This means that $Q$ is increasing. 
The inequality $\dot{\Sigma} \leqslant-C Q$ for a constant $C>0$ then shows that the solution must leave the region of interest in finite time, so that there can be no $\omega$-limit point with $\Sigma_{+} \geqslant \frac{1}{2}$.

For convenience an interior solution, which does not tend to $P_{4}$ as $\tau \rightarrow-\infty$ will be called exceptional. Thus lemma 3.1 says that an exceptional solution cannot intersect the region $\Sigma_{+}>\frac{1}{2}$.

Lemma 3.2. The $\alpha$-limit set of an exceptional solution cannot intersect the boundary at any point except $P_{3}$. If it does intersect the boundary at $P_{3}$ it belongs to case $(b)$ of theorem 3.1 as $\tau \rightarrow-\infty$. The $\omega$-limit set of any interior solution cannot intersect the boundary at all.

Proof. Let $\left(r, \Sigma_{+}\right)$be a point of the $\alpha$-limit set of an exceptional solution which lies on the boundary. If $r=\infty$ then the whole orbit passing through that point belongs to the $\alpha$-limit set. This implies that the solution must intersect the region $\Sigma_{+}>\frac{1}{2}$, a contradiction. Thus, in fact, $r<\infty$. If $\Sigma_{+}=-1$ then all points with $\Sigma_{+}=-1$ must be in the $\alpha$-limit set, in particular $P_{5}$. By lemma A.2 of the appendix, it follows that a point of the centre manifold of $P_{5}$ lies in the $\alpha$-limit set. However, this centre manifold is given by $r=\infty$ and so we again obtain a contradiction. Hence $\Sigma_{+}>-1$. If $r=0$ and $\Sigma_{+}<\frac{1}{2}$ then all points satisfying these conditions must be in the $\alpha$-limit set, in particular $P_{2}$. However, then an application of lemma A.1 of the appendix leads to a contradiction. Thus no point on the boundary other than $P_{3}$ is possible. A further application of lemma A.1 shows that in this case the solution must lie on the unstable manifold of $P_{3}$. In a similar way it is possible to show that if any point of the boundary belonged to the $\omega$-limit set of an interior solution then some point with $\Sigma_{+}>\frac{1}{2}$ would do so. However, we know from lemma 3.1 that this is impossible.

Proof of theorem 3.1. First the Poincaré-Bendixson theorem will be applied to the restriction of the Bianchi I system to the interior with the point $P_{1}$ removed. In general the $\alpha$-and $\omega$-limit sets of an orbit of a dynamical system can be very complicated, but in two dimensions (and the Bianchi I system is two dimensional) things are a lot simpler. Complicated situations are still possible and these play an important role in Hilbert's 16th problem (see e.g. [2], p 104). However, many pathologies are ruled out by the Poincaré-Bendixson theorem, which is stated in the appendix (theorem A.2).

Given an interior solution, suppose that $P_{1}$ does not belong to the $\omega$-limit set. By lemma 3.2 no point of the boundary belongs to the $\omega$-limit set either. Since $P_{1}$ is a hyperbolic sink it follows that there must be a neighbourhood of $P_{1}$ which does not intersect the $\omega$-limit set. Thus the solution remains in a compact set of the interior with the point $P_{1}$ removed as $\tau \rightarrow \infty$. Then theorem A.2 implies the existence of a non-stationary periodic orbit of the Bianchi I system. In fact, the existence of periodic solutions of the Bianchi I system can be ruled out by the presence of a Dulac function. (For a discussion of this concept see [19].) Define a function $F(r)$ by

$$
F(r)=\int f_{0}\left(p_{i}\right)\left(r^{2} p_{1}^{2}+p_{2}^{2}+p_{3}^{2}\right)^{1 / 2} \mathrm{~d} p_{1} \mathrm{~d} p_{2} \mathrm{~d} p_{3} .
$$

Then $Q=(r / F) F^{\prime}$. For $r>0$ and $\left|\Sigma_{+}\right|<1$ let

$$
G\left(r, \Sigma_{+}\right)=r^{-1} F^{1 / 2}\left(1-\Sigma_{+}^{2}\right)^{-3 / 2}
$$

and denote the vector field defining the Bianchi I system by $X$. Then $\operatorname{div}(G X)$ is negative. In fact, it is a constant multiple of $r^{-1} F^{1 / 2}\left(1-\Sigma_{+}^{2}\right)^{-1 / 2}\left(2-\Sigma_{+}\right)$. This means that $G$ is a Dulac function. It follows that the Bianchi I system has no periodic solutions. It can be concluded that $P_{1}$ does lie in the $\omega$-limit set. However, since $P_{1}$ is a hyperbolic sink, this implies, via the 
Hartman-Grobman theorem ( $\mathrm{cf}$ theorem A.1), that the $\omega$-limit set consists of $P_{1}$ alone, which proves the first part of the theorem.

To prove the remainder of the theorem we can assume without loss of generality that the solution is exceptional and that it does not lie on the unstable manifold of $P_{3}$. If $P_{1}$ were not in the $\alpha$-limit set then we would obtain a contradiction by the Poincaré-Bendixson theorem and the absence of periodic orbits. Hence $P_{1}$ must belong to the $\alpha$-limit set, and since $P_{1}$ is a hyperbolic sink, the only possibility left is case (a) of the theorem.

The conclusion of this theorem can be summarized in words as follows. All solutions isotropize in the expanding direction. The initial singularity is generically a cigar singularity but there are exceptional cases where it is a barrel or point singularity. (For this terminology see [19], p 30.) Note for comparison that if the Vlasov equation is replaced by the Euler equation for a fluid satisfying a physically reasonable equation of state then there are no barrel singularities and all solutions which are not isotropic have cigar or pancake singularities (see [14]). The pancake singularities are as common as the cigar singularities. In particular, this means that for fluid solutions of Bianchi type I cigar singularities are not generic. All solutions isotropize in the expanding direction. Note that the 'reasonable' equations of state include those of the form $p=k \rho$ with $0 \leqslant k<1$. The solutions of the Einstein-Vlasov equations approach an isotropic fluid solution with equation of state $p=\frac{1}{3} \rho$ in the sense that the tracefree part of the spatial projection $T_{i j}$ of the energy-momentum tensor divided by the energy density $\rho$ approaches zero, while $\operatorname{tr} T / \rho=1$. The latter relation is always true for kinetic theory with massless particles and for a radiation fluid (equation of state $p=\frac{1}{3} \rho$ ).

\section{Other class A models}

This section is concerned with the models of class A, as described by the system (7). Only limited statements will be made about types VIII and IX. Even in the a priori simpler case of a perfect fluid with a linear equation of state it is difficult to analyse LRS models of type VIII and IX. (For information on what is known about that case, see [17,18] and section 8.5 of [19].) A major difficulty is that in these cases the domain of definition of the dynamical system is non-compact. This allows the possibility that there may be anomalous solutions similar to those encountered in [16]. Type I was analysed in the previous section and we will see that the analysis of type $\mathrm{VII}_{0}$ can be reduced to that case in a relatively straightforward way. The most interesting results are obtained for Bianchi type II.

We start with a theorem on Bianchi type $\mathrm{VII}_{0}$, which is a close analogue of theorem 3.1.

Theorem 4.1. If a smooth non-vacuum reflection-symmetric LRS solution of Bianchi type VII ${ }_{0}$ of the Einstein-Vlasov equations for massless particles is represented as a solution of (7) with $N_{1}=0$ then for $\tau \rightarrow \infty$ the pair $\left(r, \Sigma_{+}\right)$converges to $\left(r_{0}, 0\right)$ while $N_{2}$ increases without limit. $N_{2}$ tends to zero as $\tau \rightarrow-\infty$ while the pair $\left(r, \Sigma_{+}\right)$either

(a) converges to $P_{1}$ and in that case it stays for all time at the point $P_{1}$ or

(b) converges to the point $P_{3}$ and belongs to the unstable manifold of $P_{3}$ or

(c) converges to $P_{4}$.

All of these cases occur, and (c) is the generic case in the sense that it occurs for an open dense set of initial data.

Proof. When $N_{1}=0$ the third equation in (7) becomes

$$
\dot{N}_{2}=\frac{1}{3}\left(1+\Sigma_{+}\right)^{2} N_{2}
$$


while the equations for $\Sigma_{+}$and $r$ do not involve $N_{2}$. The latter equations form a subsystem, which is identical to the equations for Bianchi type I, so that the situation is again as in figure 1 . The qualitative behaviour of their solutions has been analysed in theorem 3.1. All that remains to be done is then to put that information into equation (22) and read off the behaviour of $N_{2}$. The expression $\left(1+\Sigma_{+}\right)^{2}$ is strictly positive for a non-vacuum solution of type $\mathrm{VII}_{0}$, due to (10). Moreover, it is bounded by 4 . Thus the solution has the property that the sign of $N_{2}$ remains constant and the solution exists globally in $\tau$. It is also clear that $N_{2} \rightarrow \infty$ as $\tau \rightarrow \infty$ and that $N_{2} \rightarrow 0$ as $\tau \rightarrow-\infty$.

There is a simple explanation for the close relation between the Bianchi I and Bianchi $\mathrm{VII}_{0}$ solutions. They are, in fact, the same spacetimes parametrized in two different ways. The full four-dimensional isometry group has a subgroup of Bianchi type I and a one-parameter family of subgroups of Bianchi type $\mathrm{VII}_{0}$.

Next we turn to the solutions of type II. It will be shown that the stationary points of (the compactification of) (7) which lie in the closure of $S_{2}$ are the points $P_{1}, \ldots, P_{6}$ which we know already together with one additional point $P_{7}$, which has coordinates $\left(\infty, \frac{2}{5} \sqrt{2}, \frac{1}{5}\right)$ (see figure 2). The corresponding distributional solution of the Einstein-Vlasov equations will be discussed in detail below.

Theorem 4.2. If a smooth non-vacuum reflection-symmetric LRS solution of Bianchi type II of the Einstein-Vlasov equations for massless particles is represented as a solution of (7) with $N_{2}=0$ then for $\tau \rightarrow \infty$ the solution converges to $P_{7}$. For $\tau \rightarrow-\infty$ either:

(a) the solution converges to $P_{1}$ or

(b) the $\alpha$-limit set of the solution consists of the points $P_{2}, P_{4}, P_{5}$ and $P_{6}$ together with the orbits connecting $P_{2}$ to $P_{5}, P_{5}$ to $P_{6}$ and $P_{6}$ to $P_{4}$ in the set $N_{1}=0$ and the stable manifold of $P_{4}$ which connects $P_{4}$ with $P_{2}$ via the vacuum boundary. In particular, $\liminf \operatorname{in}_{\tau \rightarrow-\infty} \Sigma_{+}=-1, \limsup _{\tau \rightarrow-\infty} \Sigma_{+}=+1, \liminf _{\tau \rightarrow-\infty} r(\tau)=-\infty$ and $\lim \sup _{\tau \rightarrow-\infty} r(\tau)=\infty$.

Both of these cases occur and $(b)$ is the generic case.

This theorem shows that while models of Bianchi type II have simple behaviour in the expanding phase, all tending to a single attractor, the behaviour near the initial singularity is in general oscillatory, and quite different from the Bianchi type I case. Note also that the models of type II do not isotropize as $\tau \rightarrow \infty$, which is another important difference from the type I models.

A first important step in proving theorem 4.2 is to use the identity

$$
\partial / \partial \tau\left(r^{4 / 3} N_{1}\right)=r^{4 / 3} N_{1}\left[-\frac{1}{4} N_{1}\left(N_{1}-4 N_{2}\right)+\frac{1}{3}\left(1-\Sigma_{+}^{2}\right)+\frac{2}{3} \Sigma_{+}^{2}\right]
$$

which holds for any solution of (7). This is done in the following lemma.

Lemma 4.1. For any solution in the interior of $S_{2}$ the following statements hold. As $\tau \rightarrow \infty r$ tends to $\infty$. Either the $\alpha$-limit set is contained in the set $r=0$ or it contains one of the points $P_{1}, \ldots, P_{6}$.

Proof. For a non-vacuum solution a strict inequality holds in (10) and hence $r^{4 / 3} N_{1}$ is strictly increasing where it is non-zero. This means that as long as $r$ is finite and the Bianchi type is II this quantity is always increasing. Since $S_{2}$ is compact, solutions exist globally in $\tau$. As $\tau$ tends to plus or minus infinity the solution must go to the boundary of $S_{2}$. Equation (23) shows that if $r^{4 / 3} N_{1}$ tends to a finite non-zero limit in either time direction then $\Sigma_{+}^{2}$ is integrable 

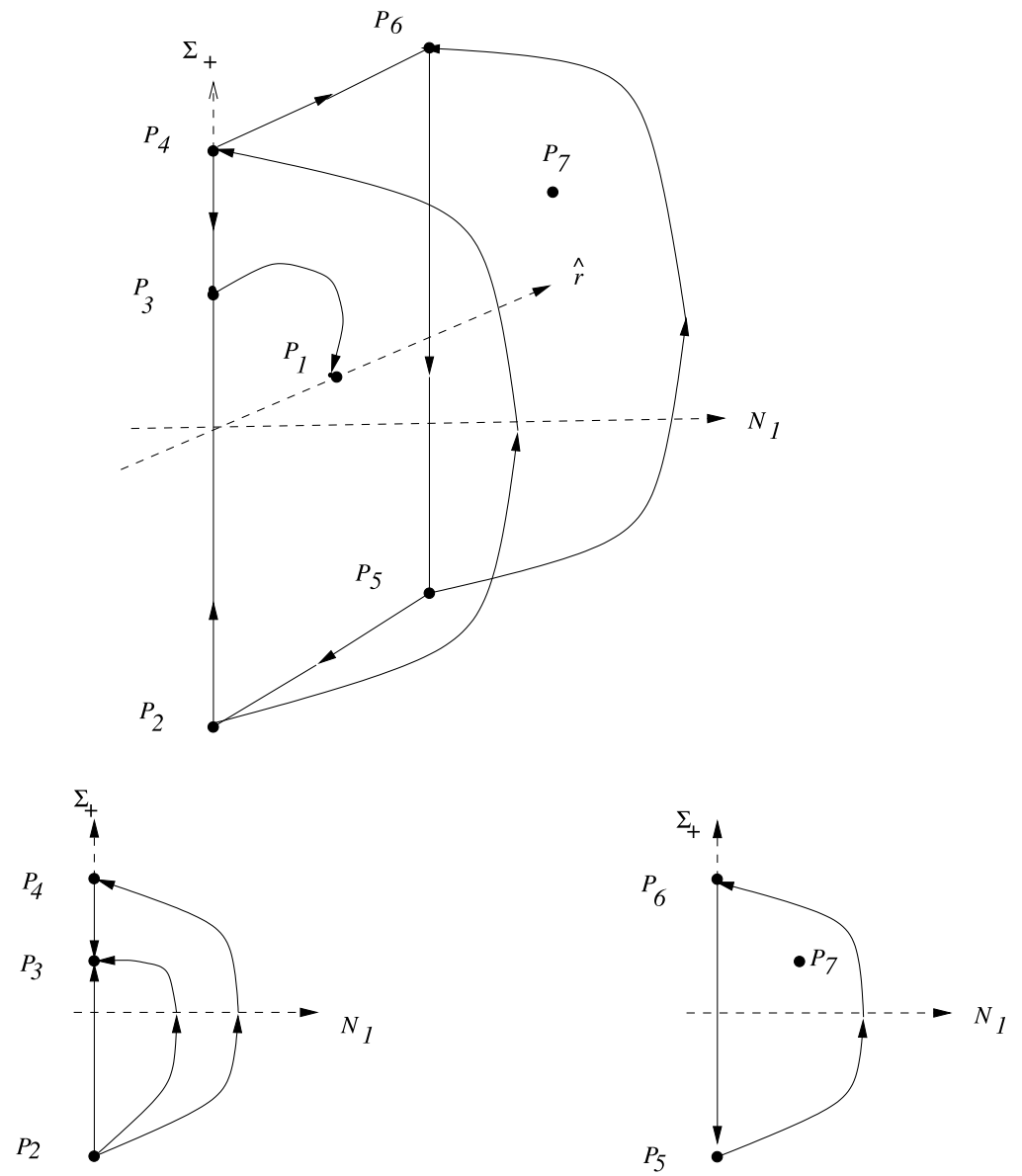

Figure 2. The $\left(\hat{r}, \Sigma_{+}, N_{1}\right)$-space and the fixed points for Bianchi type II; the lower two figures show the phase portraits on the end-faces $\hat{r}=0,1$.

on a half-infinite time interval. The derivative of this quantity is bounded and these two facts together imply that it must tend to zero in the limit. The same argument applies to the quantity appearing in (10) and so it must also tend to zero in the limit. Under these conditions $N_{1} \rightarrow 2 / \sqrt{3}$ and $\dot{\Sigma}_{+} \rightarrow \frac{4}{3}$, a contradiction. It can be concluded that $\lim _{\tau \rightarrow \infty}\left(r^{4 / 3} N_{1}\right)(\tau)=\infty$ and $\lim _{\tau \rightarrow-\infty}\left(r^{4 / 3} N_{1}\right)(\tau)=0$. From the first of these statements and the boundedness of $N_{1}$ it follows that $r$ tends to $\infty$ as $\tau$ tends to $\infty$. The fact that $r^{4 / 3} N_{1}$ tends to zero in the contracting direction implies that the $\alpha$-limit set is contained in the union of the sets $r=0$ and $N_{1}=0$. Suppose the $\alpha$-limit set contains some point for which $r \neq 0$. This must belong to the Bianchi I set. Thus the $\alpha$-limit set contains a solution of Bianchi type I. Using theorem 3.1, we conclude that the $\alpha$-limit set contains one of the points $P_{1}, \ldots, P_{6}$.

The next lemma gives information about the nature of the stationary points on $S_{2}$. We already know from lemma 4.1 that these stationary points can only occur for $N_{1}=0, r=0$ or $\infty$. The stationary points $P_{1}, \ldots, P_{6}$ will be investigated first. The equations for $r=0$ and $\infty$ will be studied in detail later. 
Lemma 4.2. The stationary points $P_{1}, \ldots, P_{4}$ and $P_{6}$ of the restriction of the system (7) to $S_{2}$ are hyperbolic saddles, while $P_{5}$ is degenerate. The stable manifold of $P_{1}$ is given by $N_{1}=0$. The stable and unstable manifolds of $P_{2}$ are given by $\Sigma_{+}=-1, N_{1}=0$ and $r=0$, respectively. The stable manifold of $P_{3}$ is given by $r=0$. The stable and unstable manifolds of $P_{4}$ are given by $r=0, N_{1}^{2}=\frac{4}{3}\left(1-\Sigma_{+}^{2}\right)$ and $N_{1}=0$, respectively. The stable and unstable manifolds of $P_{6}$ are given by $N_{1}^{2}=\frac{4}{3}\left(1-\Sigma_{+}^{2}\right)$ and $r=\infty, N_{1}=0$, respectively. The unstable manifold of $P_{5}$ is given by $N_{1}^{2}=\frac{4}{3}\left(1-\Sigma_{+}^{2}\right)$. The set $r=\infty, N_{1}=0$ is a centre manifold for $P_{5}$.

Proof. All that needs to be done is to compute the linearizations of the system about the given points and to note that the manifolds named in the statement of the theorem are all invariant. Linearizing the restriction of (7) to $N_{2}=0$, and setting $N_{1}=0$ in the result, gives the system (a bar denotes a linearized quantity):

$$
\begin{aligned}
& \mathrm{d} \bar{r} / \mathrm{d} \tau=\Sigma_{+} \bar{r}+r \bar{\Sigma}_{+} \\
& \mathrm{d} \bar{N}_{1} / \mathrm{d} \tau=\frac{1}{3}\left(1-4 \Sigma_{+}+\Sigma_{+}^{2}\right) \bar{N}_{1} \\
& \mathrm{~d} \bar{\Sigma}_{+} / \mathrm{d} \tau=\left[-\frac{1}{3}\left(1+\Sigma_{+}-3 \Sigma_{+}^{2}\right)+\Sigma_{+} Q(r)\right] \bar{\Sigma}_{+}-\frac{1}{3} Q^{\prime}(r)\left(1-\Sigma_{+}^{2}\right) \bar{r} .
\end{aligned}
$$

The linearization about $P_{1}$ has eigenvalues $\frac{1}{3}$ and $-\frac{1}{6} \pm \frac{1}{2} \sqrt{\frac{1}{9}-\frac{4}{3} r_{0} Q^{\prime}\left(r_{0}\right)}$. The invariant subspace of the linearization corresponding to the eigenvalues with negative real parts is the tangent space to $N_{1}=0$. The linearizations about $P_{2}, P_{3}$ and $P_{4}$ are diagonal with diagonal entries $(-1,2,1),\left(\frac{1}{2},-\frac{1}{4},-\frac{1}{4}\right)$ and $\left(1,-\frac{2}{3}, \frac{1}{3}\right)$, respectively. Since $P_{5}$ and $P_{6}$ lie at $r=\infty$, we must change to the coordinate $\tilde{r}$ to study the linearizations at these points. They are diagonal with diagonal elements $(1,2,0)$ and $\left(-1,-\frac{2}{3}, \frac{4}{3}\right)$.

Next the limiting systems for $r=0$ and $\infty$ will be examined.

Lemma 4.3. Consider the restriction of the system (7) to the set given by the equations $N_{2}=r=0$. For any solution which does not belong to the vacuum boundary and which does not satisfy $N_{1}=0$, the $\alpha$-limit set is the point $P_{2}$ and the $\omega$-limit set is the point $P_{3}$.

Proof. First it will be shown that the solution cannot be stationary. The equation for $\Sigma_{+}$shows that $\dot{\Sigma}_{+}>0$ if $\Sigma_{+}<\frac{1}{2}$. Thus at a stationary point $\Sigma_{+} \geqslant \frac{1}{2}$. On the other hand, the equation for $N_{1}$ shows that at a stationary point

$$
\left(\Sigma_{+}-2\right)^{2}=3+N_{1}^{2} \geqslant 3
$$

Using the fact that $\Sigma_{+} \leqslant 1$ it follows that $\Sigma_{+} \leqslant 2-\sqrt{3}<\frac{1}{2}$. Next, it follows from theorem 3.1 on p 150 of [7] that the solution cannot be periodic. It can be concluded using the PoincaréBendixson theorem (theorem A. 2 of the appendix) that the $\alpha$-and $\omega$-limit sets are contained in the boundary of the region. The behaviour of solutions on the boundary is easily determined. The nature of the stationary points on the boundary can be read off from lemma 4.2. $P_{2}$ is a hyperbolic source, $P_{3}$ is a hyperbolic sink and $P_{4}$ is a hyperbolic saddle. The last fact means, using lemma A.1 of the appendix, that $P_{4}$ cannot be in the $\alpha$ - or $\omega$-limit set unless $P_{2}$ or $P_{3}$ is also. Thus it can be concluded that the $\alpha$ - and $\omega$-limit sets must contain either $P_{2}$ or $P_{3}$ and then the conclusion follows easily.

The system given by $N_{2}=0$ and $r=\infty$ is more complicated.

Lemma 4.4. Consider the restriction of the system (7) to the set given by the equations $N_{2}=0$ and $r=\infty$. For any solution which does not belong to the vacuum boundary and which does not satisfy $N_{1}=0$, the $\alpha$-limit set consists of all points, which are either on the vacuum boundary or satisfy $N_{1}=0$. The $\omega$-limit set is the point $P_{7}$. 
Proof. Define a function $Z$ by

$$
Z=N_{1}^{1 / 2}\left[-\frac{1}{2} N_{1}^{2}+\frac{2}{3}\left(1-\Sigma_{+}^{2}\right)\right]^{3 / 4}\left(1-\frac{1}{5} \Sigma_{+}\right)^{-2} .
$$

This function is well defined and continuous on $S_{2}$ and smooth away from $N_{1}=0$ and $-\frac{1}{2} N_{1}^{2}+\frac{2}{3}\left(1-\Sigma_{+}^{2}\right)=0$. Its derivative is given by

$\partial_{\tau} Z=\frac{1}{10} Z\left(1-\frac{1}{5} \Sigma_{+}\right)^{-1}\left[\frac{1}{3}\left(5 \Sigma_{+}-1\right)^{2}+\left(-3 N_{1}^{2}+\Sigma_{+}^{2}-15 \Sigma_{+}+4\right)(1-Q)\right]$.

The restriction of $Z$ to the set $r=\infty$ is non-decreasing along solutions as a consequence of (27). Moreover, it is strictly increasing unless $\Sigma_{+}=\frac{1}{5}$. When $\Sigma_{+}=\frac{1}{5}$ it follows from (7) that $\dot{\Sigma}_{+} \neq 0$ unless $N_{1}=\frac{2}{5} \sqrt{2}$. Thus apart from the stationary solution at the point $P_{7}$ with coordinates $\left(\infty, \frac{2}{5} \sqrt{2}, \frac{1}{5}\right)$, the function $Z$ is strictly increasing along any solution with $r=\infty$. It follows that $P_{7}$ is the $\omega$-limit point of all solutions. The function $Z$ attains its minimum precisely on the boundary of the region where the system is defined and hence the $\alpha$-limit set of any solution is contained in this boundary. The only stationary points on the boundary are $P_{5}$ and $P_{6}$. From lemma 4.2 it follows that both are saddle points of this system. $\left(P_{5}\right.$ is degenerate while $P_{6}$ is non-degenerate.) This suffices to show, using lemmas A.1 and A.2 of the appendix, that the $\alpha$-limit set consists of the entire boundary.

Proof of theorem 4.2. By lemma 4.1 the $\omega$-limit set of any solution consists of points with $r=\infty$. Then lemma 4.4 shows that either $P_{7}$ belongs to the $\omega$-limit set or that the $\omega$-limit set consists entirely of points with $r=\infty$ and $N_{1}=0$ or $-\frac{1}{2} N_{1}^{2}+\frac{2}{3}\left(1-\Sigma_{+}^{2}\right)=0$. A calculation of the linearization of (7) around $P_{7}$ shows that this point is a hyperbolic sink. Hence if $P_{7}$ belongs to the $\omega$-limit set this set consists of $P_{7}$ alone. It remains to rule out the other possibility where the solution has an $\omega$-limit point on the boundary of the intersection of $S_{2}$ with $r=\infty$. In that case lemma A.1 applied to the point $P_{6}$ and lemma A.2 applied to the point $P_{5}$ show that the $\omega$-limit set contains the whole of this boundary. It will now be shown using (27) that this leads to a contradiction. There exist $\delta_{1}>0$ and $M>0$ such that if $\left|\Sigma_{+}-\frac{1}{5}\right|>\delta_{1}$ and $r>M$ the right-hand side of (27) is positive. This is because the first term dominates the second. By reducing $\delta_{1}$ and increasing $M$ if necessary it can be ensured that there exist positive constants $\eta_{1}, \eta_{2}$ and $\delta_{2}$ such that $Z^{-1} \partial_{\tau} Z$ can be bounded below by $\eta_{1}$ as long as $\left|\Sigma_{+}-\frac{1}{5}\right|>\delta_{1}$ and $r>M$ and $\dot{\Sigma}_{+}>\eta_{2}$ for $\left|\Sigma_{+}-\frac{1}{5}\right|<\delta_{1},\left|N_{1}-\frac{2}{5} \sqrt{2}\right|>\delta_{2}$ and $r>M$. Finally, given $\eta_{3}>0$ there exists $\delta_{3}>0$ so that $\dot{\Sigma}<\eta_{3}$ for $|\Sigma|>1-\delta_{3}$ and $r>M$. At sufficiently late times the solution lies in the region $r>M$. Moreover, under the present assumption on the $\omega$-limit set it cannot enter the neighbourhood of $P_{7}$ defined by $\delta_{1}$ and $\delta_{2}$. Each time it crosses the strip defined by $\left|\Sigma_{+}-\frac{1}{5}\right| \leqslant \delta_{1}$ at a sufficiently late time it must enter the region $\Sigma_{+}>1-\delta_{3}$ before it can return to the strip. It must spend a long time in the region $\Sigma_{+}>1-\delta_{3}$ (due to the smallness of $\eta_{3}$ ). This time can be bounded below by $C \eta_{3}^{-1}$ for a constant $C>0$. During that time $\log Z$ must increase by at least $C\left(\eta_{1} / \eta_{3}\right)$. On the other hand, $\log Z$ can only decrease while it is in the strip. It stays there for a time at most $\delta_{1} / \eta_{2}$ and can decrease by at most $C \delta_{1} / \eta_{2}$. Thus the net change of $Z$ for each time it enters the strip is at least $C\left(\eta_{1} / \eta_{3}-\delta_{1} / \eta_{2}\right)$. If $\eta_{3}$ is chosen small enough this will be bounded below by a positive quantity. Since the solution must, under the given assumptions, enter the strip infinitely often, this gives a contradiction. The proof of the statement about the $\omega$-limit set is now complete.

Suppose that the $\alpha$-limit set contains a point with $r=0$ and $N_{1} \neq 0$. Then by lemma 4.3 it contains $P_{2}$ and $P_{3}$ or $P_{4}$. Lemma 4.1 then shows that at least one of $P_{1}, \ldots, P_{6}$ is contained in the $\alpha$-limit set. If the $\alpha$-limit set contains $P_{1}$ then either the solution lies in the unstable manifold of $P_{1}$, which gives case (a) of the theorem, or the $\alpha$-limit set contains points on 
that unstable manifold other than $P_{1}$ itself. However, since these satisfy neither $N_{1}=0$ or $r=0$ this is a contradiction. If the $\alpha$-limit set contained points with $N_{1}=0$ with $r$ finite and $\left|\Sigma_{+}\right|<1$ it would contain $P_{1}$, leading once again to a contradiction. If it contains $P_{4}$ it follows from lemma A.1 and what has just been said that it must contain either $P_{3}$ or $P_{6}$. It must also contain $P_{2}$. However, if it contained $P_{3}$ it would, by another application of the same lemma contain $P_{1}$, a contradiction. On the other hand, if it contains $P_{2}$ it must contain $P_{4}$ and $P_{5}$. If it contains $P_{5}$ it must contain $P_{6}$ and vice versa, by lemmas A.1 and A.2. On the other hand, lemma A.1 shows that if the $\alpha$-limit set contains $P_{5}$ or $P_{6}$ it must contain $P_{2}$ or $P_{4}$. It also follows from these applications of the lemmas of the appendix that the relevant connecting orbits are contained in the $\alpha$-limit sets.

Now a spacetime corresponding to the point $P_{7}$ will be determined (in figure 2, this spacetime follows a straight line at constant $\left(\Sigma_{+}, N_{1}\right)$ into $\left.P_{7}\right)$. From equation (19) it follows that $\operatorname{tr} k=H_{0} \mathrm{e}^{-3 / 5 \tau}$. Putting this in the equation relating $t$ and $\tau$ shows that $\operatorname{tr} k=-\frac{5}{3} t^{-1}$. Putting this in the third equation of (6) gives $b=b_{0} t^{2 / 3}$. Equation (5) implies that $a=a_{0} t^{1 / 3}$. Finally, the second equation of (6) leads to the relation $a_{0}=\frac{2}{5} \sqrt{2} b_{0}^{2}$. Choosing an explicit representation of a Bianchi type II frame leads to the metric

$$
\mathrm{d} s^{2}=-\mathrm{d} t^{2}+\frac{8}{9} B^{2} t^{2 / 3}(\mathrm{~d} x+z \mathrm{~d} y)^{2}+B t^{4 / 3}\left(\mathrm{~d} y^{2}+\mathrm{d} z^{2}\right)
$$

where $B$ is a constant. This metric is invariant under the homothety $t \mapsto A t, x \mapsto A^{2 / 3} x$, $y \mapsto A^{1 / 3} y, z \mapsto A^{1 / 3} z$. It follows that $t \partial / \partial t+\frac{2}{3} x \partial / \partial x+\frac{1}{3}(y \partial / \partial y+z \partial / \partial z)$ is a homothetic vector field and that this metric is self-similar. It satisfies the Einstein equations with an energy-momentum tensor whose only non-vanishing components are $\rho$ and $T_{11}$. These two are equal and are proportional to $t^{-2}$. This can be interpreted as a distributional solution of the Einstein-Vlasov equations with massless particles where the distribution function is of the form $f\left(p_{1}, p_{2}, p_{3}\right)=f_{1}\left(p_{1}\right) \delta\left(p_{2}\right) \delta\left(p_{3}\right)$. (Note that a distributional $f$ of this kind defines a dynamical system just as a smooth $f$ does so that the solution can be represented in figure 2.) The exact form of the function $f_{1}$ is unimportant. Only the integrals $\int f_{1}\left(p_{1}\right) p_{1} \mathrm{~d} p_{1}$ and $\int f_{1}\left(p_{1}\right) p_{1}^{2} \mathrm{~d} p_{1}$ influence the energy-momentum tensor. Related to this fact is that the same spacetime can be interpreted as a solution of the Einstein equations coupled to two streams of null dust moving in opposite senses in the $x^{1}$-direction. This corresponds to choosing $f_{1}=\left(\delta\left(p_{1}\right)+\delta\left(-p_{1}\right)\right)$ instead of a smooth function. The sum of two Dirac measures is necessary to preserve the reflection symmetry. This spacetime has previously been considered by Dunn and Tupper [5] in the context of cosmological models with electromagnetic fields, although it had to be rejected for their purposes since no consistent electromagnetic field existed.

The monotonic function $Z$ which plays a crucial role in the proof of theorem 4.2 is rather complicated and so is unlikely to be found by trial and error. We found it by means of a Hamiltonian formulation of the equations for $r=\infty$. Once the function was found for $r=\infty$ it was extended so as to be independent of $r$. In developing the Hamiltonian formulation we followed the treatment of Uggla in chapter 10 of [19]. A key point is that the energy density of a distributional solution of the Einstein-Vlasov system where the pressure is concentrated in one direction can be related in a simple way to $r$. The function $Z$ is the Hamiltonian for the (time-dependent) Hamiltonian system. It was also the construction of $Z$ which led us to discover the self-similar solution corresponding to the point $P_{7}$.

The picture obtained in theorem 4.2 is quite different from that seen in LRS Bianchi type II solutions with a perfect fluid with a linear equation of state as the matter model (see [19], chapter 6). There generic solutions are approximated near the singularity by a vacuum solution (the type II NUT solution) and there is no oscillatory behaviour. In the expanding direction the 
fluid solutions are also all asymptotic to a self-similar solution (the Collins-Stewart solution), but this solution has a different ratio of shear to expansion from the solution corresponding to the point $P_{7}$. Moreover, the pressure is highly anisotropic in the latter solution.

\section{Kantowski-Sachs and Bianchi type III models}

In this section information will be obtained on Kantowski-Sachs models and models of Bianchi type III which is as complete as that obtained on models of Bianchi type I in section 3.

Theorem 5.1. If a smooth non-vacuum reflection-symmetric Kantowski-Sachs-type solution of the Einstein-Vlasov equations for massless particles is represented as a solution of (11) with $\epsilon=1$ then for $\tau \rightarrow-\infty$ either

(a) it converges to $P_{1}$

(b) it converges to the point $P_{3}$ and it belongs to the unstable manifold of $P_{3}$ or

(c) it converges to $P_{4}$.

(d) All of these cases occur, and (c) is the generic case in the sense that it occurs for an open dense set of initial data.

Proof. The inequality $\partial_{\tau} B \geqslant \frac{1}{4} B$ shows that $B$ decreases towards the past. It follows that as $\tau$ decreases the solution remains in a compact set and hence that the solution exists for all sufficiently negative $\tau$. Using the inequality again shows that $B \rightarrow 0$ exponentially as $\tau \rightarrow-\infty$ and the $\alpha$-limit set lies in the set $B=0$. The latter can be identified with the Bianchi I system. The $\alpha$-limit set contains the image of a solution of the Bianchi I system and hence, by theorem 3.1 contains either $P_{1}$ or some point of the boundary of the Bianchi I system. Each of the stationary points $P_{1}, \ldots, P_{6}$, considered as stationary points of (11), has a linearization, which differs from its linearization within the Bianchi I system by the addition of an extra eigenvector with a positive eigenvalue. It can be concluded from this that $P_{4}$ is a hyperbolic source. Moreover, by lemmas A.1 and A.2, if any point of the boundary other than $P_{3}$ lies in the $\alpha$-limit set, then $P_{4}$ must also lie in the $\alpha$-limit set. Hence in this case the $\alpha$-limit set consists of $P_{4}$ alone. Moreover, if $P_{3}$ lies in the $\alpha$-limit set then the solution must lie on its unstable manifold. The only remaining possibility is that the $\alpha$-limit set consists of $P_{1}$ alone, and that the solution lies on the unstable manifold of $P_{1}$.

No statement is made here about the behaviour as $\tau \rightarrow \infty$. In fact, any solution of (11) with $\epsilon=1$ tends to infinity in finite time. However, this is not a problem from the point of view of understanding the spacetime. It is known that the Kantowski-Sachs models recollapse [4]. Thus there is no infinitely expanding phase and a final singularity looks like the time reverse of an initial singularity. One interesting question which we do not attempt to tackle here is whether there is an interesting correlation between the behaviour near the initial and final singularities. For each individual singularity the picture is essentially identical to that seen in the singularity of Bianchi I models. The system for a radiation fluid can be analysed in the same way, reducing the dynamics near the singularity to that of the corresponding Bianchi I system. The differences between radiation fluid and kinetic models are similar in both cases.

Theorem 5.2. If a smooth non-vacuum reflection-symmetric LRS solution of Bianchi type III of the Einstein-Vlasov equations for massless particles is represented as a solution of (11) with $\epsilon=-1$ then for $\tau \rightarrow \infty$ it converges to the point $P_{9}$ with coordinates $\left(\infty, \frac{1}{2}, \frac{1}{2}\right)$ and for $\tau \rightarrow-\infty$ either

(a) it converges to $P_{1}$ or 
(b) it converges to the point $P_{3}$ and it belongs to the unstable manifold of $P_{3}$ or

(c) it converges to $P_{4}$.

(d) All of these cases occur, and (c) is the generic case in the sense that it occurs for an open dense set of initial data.

Proof. The inequality (12) with $\epsilon=-1$ implies that a solution of (11) of Bianchi type III remains in a compact set and hence exists globally in $\tau$. The quantity $\dot{B}$ is positive in the region where $B^{2}<\frac{1}{4}+\frac{1}{12}\left(1-2 \Sigma_{+}\right)^{2}$. Call this region $G$. In the complement of the closure of $G$ the inequality $\dot{B}<0$ holds. Thus any stationary point with $B>0$ must lie on the boundary of $G$. A stationary point with a finite non-zero value of $r$ must satisfy $\Sigma_{+}=0$ and this implies that $\dot{\Sigma}=\frac{1}{3}$, a contradiction. Thus the only stationary points occur for $B=0$ (these are the well known Bianchi type I stationary points), $r=0$ or $\infty$. In fact, the only stationary points which are not of type I are those with coordinates $\left(0, \frac{1}{2}, \frac{1}{2}\right)$ and $\left(\infty, \frac{1}{2}, \frac{1}{2}\right)$. Call these $P_{8}$ and $P_{9}$, respectively (see figure 3 ).

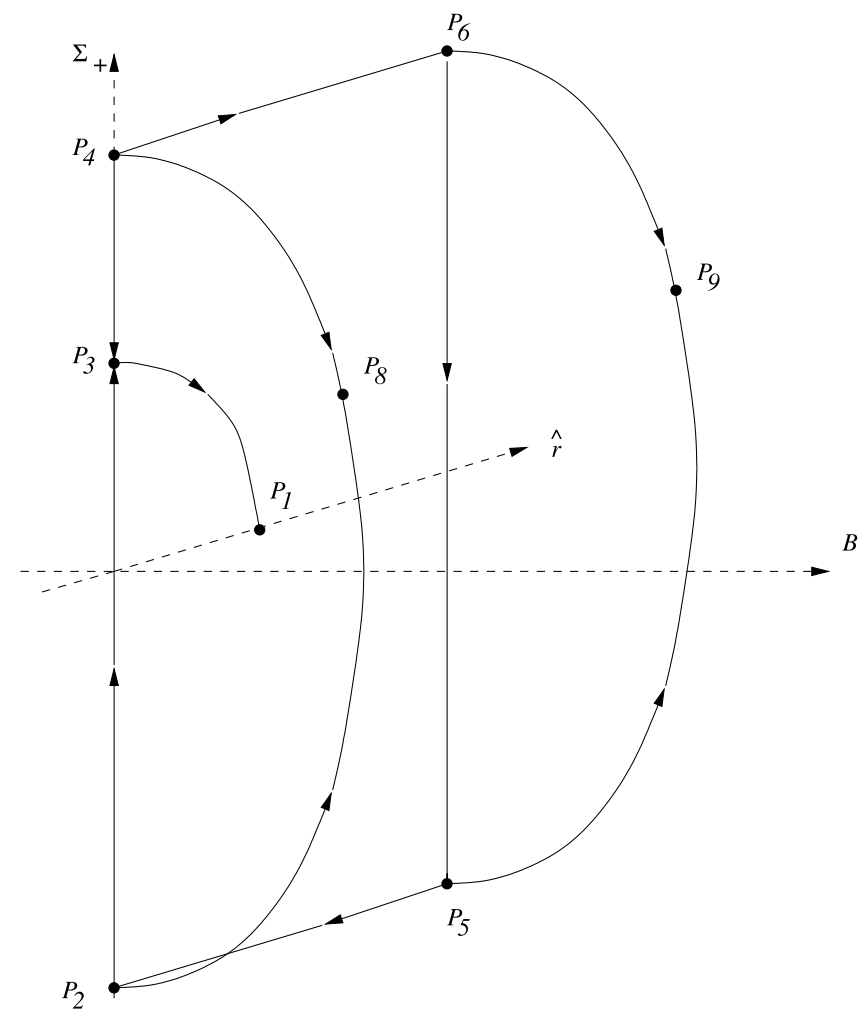

Figure 3. The $\left(\hat{r}, \Sigma_{+}, B\right)$-space and the fixed points for Bianchi type III.

The boundary of $G$ is connected and so $\dot{\Sigma}$ has a constant sign there. Checking at one point shows that this sign is positive. As a consequence, a solution can never leave $G$ as $\tau$ increases or enter $G$ as $\tau$ decreases. A solution which lies on the boundary of $G$ at some time (with $r$ non-zero and finite) must immediately enter $G$ to the future and enter the interior of its complement to the past. Consider now the behaviour of a given solution as $\tau$ decreases. If it stayed in $G$ forever then $B$ would have to increase as $\tau$ decreases. On the other hand, any $\alpha$-limit point would have to be in the boundary of $G$ due to the monotonicity properties 
of $B$. This is not consistent. Thus as $\tau$ decreases the solution must reach the boundary of $G$ and, as a consequence the interior of the complement of $G$. In the latter region $B$ is strictly monotone and so the $\alpha$-limit set must be contained in $B=0$. Then the same analysis as in the proof of theorem 5.1 shows that the solution belongs to one of the cases (a)-(c) of the theorem. Next consider the behaviour as $\tau$ increases. As $\tau$ tends to infinity the solution must tend to the boundary of $G$. If it stays in the interior of the complement of $G$ then it must tend to the boundary of $G$ as $\tau$ tends to infinity and, more precisely, to one of the points $P_{8}$ or $P_{9}$. Since $\Sigma_{+}$is positive at these points, $r \rightarrow \infty$ and so only $P_{9}$ is possible. Now suppose that the solution does meet the boundary of $G$ and hence enters $G$ itself. Then it remains in $G$ and $B$ is once again strictly monotonic. As before, it can be concluded that the solution converges to $P_{9}$ as $\tau \rightarrow \infty$.

The point $P_{9}$ corresponds to a self-similar solution of the vacuum Einstein equations much as does $P_{7}$ (this time the trajectory is the horizontal straight line in figure 3 from $P_{8}$ to $P_{9}$ ). This is the Bianchi III form of flat space (see p 193 of [19]). Once again the nature of the initial singularity is similar to that in solutions of type I. On the other hand, the final singularity is qualitatively different from any we have seen so far. In this case the solution is approximated at large times by a vacuum solution in the sense that the dimensionless quantity $\rho /(\operatorname{tr} k)^{2}$ tends to zero as $t \rightarrow \infty$. A very similar analysis applies to the system for a radiation fluid. LRS Bianchi type III fluid solutions with equation of state $p=\frac{1}{3} \rho$ behave like solutions of Bianchi type I near the initial singularity. They are approximated at large times by the same vacuum solution as in the case of kinetic theory. The approach of [8] should allow similar statements to be proved for other fluids with a linear equation of state, but this does not seem to have been worked out explicitly in the literature.

\section{Conclusions}

The above theorems show that solutions of the Einstein-Vlasov equations with high symmetry exhibit a wide variety of asymptotic behaviour near a singularity and in a phase of unlimited expansion. They can have a point singularity, barrel singularity or cigar singularity or they can show oscillatory behaviour near a singularity. In an expanding phase they can resemble a fluid solution (Bianchi type I and $\mathrm{VII}_{0}$ ), a vacuum solution (Bianchi type III) or a solution of the Einstein equations with null dust (Bianchi type II). There are notable differences in comparison with a fluid model, and this includes the radiation fluid, which is often used as an effective model of massless particles in cosmology. The most striking qualitative difference is the appearance of oscillatory behaviour in type II solutions. It is interesting to compare this with the analysis of spacetime singularities by Belinskii et al [3]. They do not say precisely what they assume about matter but it seems that they do assume, at least implicitly, that pressures cannot approach the energy density. This assumption is not necessarily satisfied in a kinetic description. The mean pressure cannot exceed one third of the energy density, but if it all concentrates in one direction the pressure in that direction can approach the energy density. This leads to a source of oscillations beyond those taken into account in [3].

While the oscillatory behaviour of cosmological models near a singularity has often been observed numerically and explained heuristically, it has rarely been captured in rigorous theorems. To our knowledge the only example where this had been done previous to theorem 4.2 of this paper is in a class of solutions of the Einstein-Maxwell equations of Bianchi type $\mathrm{VI}_{0}$ analysed in [10].

The results of this paper concern only massless particles. One may ask what would change in the results if the case of massive particles is considered. In one case the answer is known, 
namely in Bianchi type I. There the solution approaches a dust solution in the expanding phase. It is reasonable to expect that this happens more generally. As the model expands in all directions the pressures should become negligible with respect to the energy density, leading to a dust-like situation. However, the techniques necessary to prove this are not yet known. Near the initial singularity, the equations for massive particles look like those for massless particles and it may be conjectured that the behaviour near the singularity is similar in both cases. Unfortunately that has also not yet been proved.

It is interesting to note that matter seems to have the effect of making the evolution of the geometry under the Einstein equations less extreme in a phase of unlimited expansion. In Bianchi type I the vacuum solutions (Kasner solutions) are such that some spatial direction is contracting or unchanging in the expanding time direction (the time direction in which the volume is increasing). This is no longer the case when perfect fluid or kinetic matter is added, since then the model isotropizes. In type II there is no complete isotropization but it is still the case that with fluid or kinetic matter all directions are eventually expanding, in contrast to the vacuum case. The type III case is borderline, since there solutions with collisionless matter are asymptotic, in the sense of the variables used in this paper, to a vacuum solution in the expanding time direction. The vacuum solution is such that the scale factor $b$ is time independent. In the other LRS Bianchi type III vacuum spacetimes this scale factor is asymptotically constant as $t \rightarrow \infty$ and for a radiation fluid this is also the case (cf [19], p 203). On the other hand, for dust models, which also converge to the same vacuum model in terms of the Wainwright-Hsu variables, this scale factor grows without bound, although much more slowly than the other scale factors ([19], p 202). It is difficult to decide what happens in the case of collisionless matter with massless particles, since the point $P_{9}$ is a degenerate stationary point of the system (11). In the corresponding system for a radiation fluid the point with these coordinates is also a stationary point but is non-degenerate.

For the Einstein-Vlasov equations with massless particles the LRS reflection-symmetric solutions of Bianchi types I, II, III, $\mathrm{VII}_{0}$ and Kantowski-Sachs type have now been analysed as far as to give a full description of their general behaviour near the singularity and in a phase of unlimited expansion. There are still plenty of open questions related to this. What happens with LRS solutions of types VIII and IX? What happens if reflection symmetry is dropped? Does this lead to a new kind of oscillatory behaviour? What happens if the LRS condition is dropped? (This is still open even in the Bianchi I case.) Can the Hamiltonian formulation of the equations, which played an important role at one point in our arguments, usefully be applied in some of these more general cases? Answers to these questions could help to deepen our understanding of the dynamics of solutions of the Einstein equations with matter in general.

\section{Acknowledgments}

We wish to thank Malcolm MacCallum for his comments on the exact solution in section 4 . PT gratefully acknowledges the hospitality and financial support of the Max-Planck-Institut für Gravitationsphysik while this work was being done.

\section{Appendix. Some background on dynamical systems}

First some terminology will be introduced. We use the phrase 'dynamical system' as a synonym for 'system of ordinary differential equations'. The difference between the two is then only one of point of view. A stationary point of a dynamical system is a time-independent solution. An orbit of a dynamical system is the image of a solution. A point $x_{*}$ is an $\alpha$-limit point of a 
solution $x(t)$ if there is a sequence of times $t_{n}$ with $t_{n} \rightarrow-\infty$ such that $x\left(t_{n}\right) \rightarrow x_{*}$. The set of all $\alpha$-limit points of a solution is called its $\alpha$-limit set. The analogous notions of an $\omega$-limit point and an $\omega$-limit set are obtained by replacing $t$ by $-t$ in these definitions. Basic properties are that the $\alpha$-limit set is closed and that, if the solution remains in a compact set as $t \rightarrow-\infty$, it is connected. If $x_{*}$ is a point of the $\alpha$-limit set of an orbit then the orbit through $x_{*}$ lies in the $\alpha$-limit set of the original orbit. Analogous statements hold for the $\omega$-limit set. For details and proofs see, e.g., [7], chapter 7.

If $x_{0}$ is a stationary point of a dynamical system we can linearize the system about $x_{0}$. The linearized system is of the form $\mathrm{d} \tilde{x} / \mathrm{d} t=A \tilde{x}$ for a matrix $A$. Associated to $A$ is a direct sum decomposition $E_{1} \oplus E_{2} \oplus E_{3}$ where the vector spaces $E_{1}, E_{2}$ and $E_{3}$ are spanned by generalized eigenvectors of $A$ corresponding to eigenvalues with positive, zero and negative real parts, respectively. These spaces are called the unstable, centre and stable subspaces, respectively. For each of these three subspaces there is a manifold, which is tangent to the corresponding subspace at $x_{0}$ and is left invariant by the dynamical system. These manifolds are called the unstable, centre and stable manifolds, respectively. The unstable and stable manifolds are unique while the centre manifold need not be. For details see the appendix of [1].

The behaviour of solutions of a dynamical system near a stationary point is described by the reduction theorem.

Theorem A.1 (Reduction theorem). Let $x_{0}$ be a stationary point of a $C^{1}$ dynamical system. Then the system is topologically equivalent near $x_{0}$ to the Cartesian product of a standard saddle with the restriction of the flow to any centre manifold.

This theorem is proved in [9]. Topological equivalence means that there is a homeomorphism, which takes one system to the other. A standard saddle is the dynamical system on $\mathbb{R}^{n_{1}+n_{2}}$ given by $\mathrm{d} y / \mathrm{d} t=y, \mathrm{~d} z / \mathrm{d} t=-z$, where $y \in \mathbb{R}^{n_{1}}$ and $z \in \mathbb{R}^{n_{2}}$. The special case (hyperbolic case) where the centre manifold is trivial is the Hartman-Grobman theorem [7].

The next result is intuitively rather obvious, but since we do not know a published proof we will provide one here.

Lemma A.1. Let $p$ be a hyperbolic stationary point of a dynamical system which belongs to the $\alpha$-limit set of a given orbit. Then either each neighbourhood of $p$ contains a segment of the orbit, which is contained in the unstable manifold of $p$, or the $\alpha$-limit set contains a point of the stable manifold of $p$ other than $p$ itself. The analogous statement with the roles of the stable and unstable manifolds interchanged also holds.

Proof. By the reduction theorem we can assume that in a neighbourhood of $p$ the system takes the form

$$
\mathrm{d} x / \mathrm{d} t=x, \quad \mathrm{~d} y / \mathrm{d} t=-y
$$

with solution

$$
x=A \mathrm{e}^{t}, \quad y=B \mathrm{e}^{-t} .
$$

The unstable and stable manifolds are given by $y=0$ and $x=0$, respectively. Suppose that there is a neighbourhood of $p$ where there is no segment of the orbit contained in the unstable manifold. Then there exists a sequence of points $p_{n}$ on the orbit with non-vanishing $y$ coordinate, which converges to $p$. If we denote the coordinates of corresponding segments of the solution by $\left(x_{n}, y_{n}\right)$, then $y_{n}=B_{n} \mathrm{e}^{-t}$ for some $B_{n} \neq 0$. Consider now a coordinate closed ball contained in a neighbourhood of $p$ where the reduction can be carried out. As $t$ 
decreases each of the solutions $\left(x_{n}, y_{n}\right)$ must leave this ball and so must, in particular contain a point of the boundary sphere. Call the resulting sequence of points of the sphere $q_{n}$. By compactness $q_{n}$ has a subsequence converging to a point $q$. The point $q$ belongs to the $\alpha$-limit set. Now $x_{n}=A_{n} \mathrm{e}^{t}$ for a sequence with $A_{n} \rightarrow 0$. Hence the $x$ coordinate of $q$ is zero and $q$ belongs to the stable manifold of $p$. The proof in the case that the roles of the stable and unstable manifolds are interchanged is very similar, using the points where the solution exits the ball in the positive time direction.

The following variant of lemma A.1 allows a centre manifold of a certain type.

Lemma A.2. Let $p$ be a stationary point of a dynamical system, which belongs to the $\alpha$-limit set of a given orbit. Suppose that the centre manifold is one dimensional and that there is a punctured neighbourhood of $p$ in the centre manifold, which contains no stationary points and such that the solutions on the centre manifold approach $p$ as $t \rightarrow \infty$ on one side of $p$ and as $t \rightarrow-\infty$ on the other side. Suppose further that the stable manifold is trivial. The boundary between points on orbits which converge to $p$ while staying in a small neighbourhood of $p$ as $t \rightarrow-\infty$ and points on orbits which do not is the unstable manifold. The analogue of lemma A.1 holds, where the stable manifold is replaced by the half of the centre manifold on one side of the unstable manifold. This half of the centre manifold is unique.

Proof. By the reduction theorem we can assume that in a neighbourhood of $p$ the system takes the form

$$
\mathrm{d} x / \mathrm{d} t=F(x), \quad \mathrm{d} y / \mathrm{d} t=y
$$

for some function $F$ which vanishes together with its derivative at the origin, and is positive otherwise. The boundary hypersurface is given by $x=0$. The half of the centre manifold referred to in the statement of the theorem corresponds to $x<0$ and $y=0$. In the half-plane $x<0$ the system is topologically equivalent to a hyperbolic saddle and so it is possible to obtain the conclusion as in the proof of lemma A.1.

Next we state the Poincaré-Bendixson theorem. The form of this theorem which we will use is the following (cf [7], p 151):

Theorem A.2 (Poincaré-Bendixson). Let $U$ be an open subset of $\mathbb{R}^{2}$ and consider a dynamical system on $U$ without stationary points. Let $x(t)$ be a solution which exists globally and remains in a compact subset of $U$ as $t \rightarrow-\infty$. Then the $\alpha$-limit set of the given solution is a periodic orbit.

The analogous statement holds for the $\omega$-limit set. A periodic orbit is, of course, just the image of a periodic solution.

\section{References}

[1] Abraham R and Robbin J 1967 Transversal Mappings and Flows (New York: Benjamin)

[2] Arnold V I and Ilyashenko Yu S 1988 Dynamical Systems I ed D V Anosov and V I Arnold (Berlin: Springer)

[3] Belinskii V A, Khalatnikov I M and Lifschitz E M 1982 A general solution of the Einstein equations with a time singularity Adv. Phys. 13 639-67

[4] Burnett G A 1991 Incompleteness theorems for the spherically symmetric spacetimes Phys. Rev. D 43 1143-9

[5] Dunn K A and Tupper B O J 1980 Type I, II and III spatially homogeneous cosmological models with electromagnetic field Astrophys. J. 235 307-34

[6] Ehlers J 1973 Survey of general relativity theory Relativity, Astrophysics and Cosmology ed W Israel (Dordrecht: Reidel)

[7] Hartman P 1982 Ordinary Differential Equations (Boston, MA: Birkhäuser) 
[8] Hewitt C G and Wainwright J 1993 A dynamical systems approach to Bianchi cosmologies: orthogonal models of class B Class. Quantum Grav. 10 99-124

[9] Kirchgraber U and Palmer K J 1990 Geometry in the Neighborhood of Invariant Manifolds of Maps and Flows and Linearization (Harlow: Longman)

[10] Leblanc V G, Kerr D and Wainwright J 1995 Asymptotic states of magnetic Bianchi $\mathrm{VI}_{0}$ cosmologies Class. Quantum Grav. 12 513-41

[11] Lukash V N and Starobinski A A 1974 The isotropization of the cosmological expansion owing to particle production Sov. Phys.-JETP 39 742-7

[12] Maartens R and Maharaj S D 1985 Collision-free gases in spatially homogeneous spacetimes J. Math. Phys. 26 2869-80

[13] Maartens R and Maharaj S D 1990 Collision-free gases in Bianchi spacetimes Gen. Rel. Grav. 22 595-607

[14] Rendall A D 1996 The initial singularity in solutions of the Einstein-Vlasov system of Bianchi type I J. Math. Phys. 37 438-51

[15] Rendall A D 1997 An introduction to the Einstein-Vlasov system Banach Centre Publ. 41 35-68

[16] Rendall A D 1997 Global dynamics of the mixmaster model Class. Quantum Grav. 14 2341-56

[17] Uggla C and von Zur-Mühlen H 1990 Compactified and reduced dynamics for locally rotationally symmetric Bianchi type IX perfect fluid models Class. Quantum Grav. 7 1365-85

[18] Uggla C, Jantzen, R T, Rosquist K and von Zur-Mühlen H 1991 Remarks about late stage cosmological dynamics Gen. Rel. Grav. 23 947-66

[19] Wainwright J and Ellis G F R 1997 Dynamical Systems in Cosmology (Cambridge: Cambridge University Press)

[20] Wainwright J and Hsu L 1989 A dynamical systems approach to Bianchi cosmologies: orthogonal models of class A Class. Quantum Grav. 6 1409-31 